\title{
Variability of nonpathogenic influenza virus H5N3 under immune pressure
}

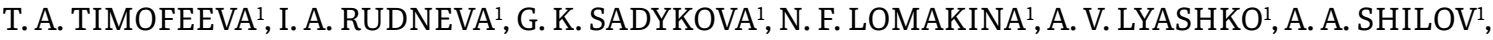 \\ O. L. VORONINA ${ }^{1}$, E. I. AKSENOVA ${ }^{1}$, N. N. RYZHOVA ${ }^{1}$, M. S. KUNDA ${ }^{1}$, M. N. ASATRYAN ${ }^{1}$, D. N. SHCHERBININ ${ }^{1}$, \\ E. B. TIMOFEEVA' ${ }^{1}$, A. A. KUSHCH ${ }^{1}$, A. G. PRILIPOV', S. E. ADAMS ${ }^{2}$, D. Y. LOGUNOV ${ }^{1}$, B. S. NARODISKY',
} A. L. GINTSBURG ${ }^{1}$

${ }^{1}$ N.F. Gamaleya National Research Center for Epidemiology and Microbiology, Ministry of Health of Russia, Gamaleya Street 18, Moscow, 123098 Russian Federation; '2University of Cambridge, Department of Pathology, Cambridge, England

Summary.-Mutations arising in influenza viruses that have undergone immune pressure may promote a successful spread of mutants in nature. In order to evaluate the variability of nonpathogenic influenza virus A/duck/Moscow/4182-C/2010(H5N3) and to determine the common epitopes between it and highly pathogenic H5N1 avian influenza viruses (HPAIV), a set of escape mutants was selected due to action of MABs specific against A/chicken/Pennsylvania/8125/83(H5N2), A/Vietnam/1203/04(H5N1) and A/duck/ Novosibirsk/56/05(H5N1) viruses. The complete genomes of escape mutants were sequenced and amino acid point mutations were determined in HA, NA, PA, PB1, PB2, M1, M2, and NP proteins. Comprehensive analysis of the acquired mutations was performed using the Influenza Research Database (https://www. fludb.org) and revealed that all mutations were located inside short linear epitopes, in positions characterized by polymorphisms. Most of the mutations found were characterized as substitutions by predominant or alternative amino acids existing in nature. Antigenic changes depended only on substitutions at positions 126, 129, 131, 145 and 156 of HA (H3 numbering). The positions 126, 145 and 156 were common for HA/H5 of different phylogenetic lineages of H5N1 HPAIV (arisen from A/goose/Guangdong/1/96) and low pathogenic American and Eurasian viruses. Additionally, mutation S145P increased the temperature of HA heat inactivation, compared to wild-type, as was proved by reverse genetics. Moreover, nonpathogenic A/ duck/Moscow/4182-C/2010(H5N3) and H5N1 HPAI viruses have the same structure of short linear epitopes in HA (145-157) and internal proteins (PB2: 186-200, 406-411; PB1: 135-143, 538-546; PA: 515-523; NP: 61-68; M1: 76-84; M2: 45-53). These facts may indicate that $\mathrm{H} 5$ wild duck nonpathogenic virus could be used as vaccine against H5N1 HPAIV.

Keywords: avian influenza virus; H5 hemagglutinin; escape mutants; genetic analysis; phenotypic properties; site-specific mutagenesis

E-mail: timofeeva.tatyana@inbox.ru; phone: +7-499-1902813. Abbreviations: AIV = avian influenza virus; GsGD = A/goose/ Guangdong/1/96(H5N1); HA = hemagglutinin; HPAIV = highly pathogenic avian influenza virus; IRD = Influenza Research Database; LPAIV = low pathogenic avian influenza virus; $\mathrm{MAB}=$ monoclonal antibody; $\mathrm{M} 1=$ matrix protein of influenza A virus; $\mathrm{M} 2$ = ion channel; $\mathrm{NA}=$ neuraminidase; $\mathrm{NP}=$ nucleoprotein; $\mathrm{PA}, \mathrm{PB} 1$ and $\mathrm{PB} 2$ = polymerase proteins; $\mathrm{SI}=$ Supplementary information

\section{Introduction}

It is likely that future flu pandemics will be caused by influenza A viruses emerging due to sporadic antigenic shift and/or reassortment between viruses, which circulate in humans and birds. The most likely candidates for pandemics are viruses with hemagglutinin $\mathrm{H1}, \mathrm{H} 2, \mathrm{H} 3, \mathrm{H} 5$, $\mathrm{H} 6, \mathrm{H} 7, \mathrm{H} 9$, and $\mathrm{H} 10$ and neuraminidase $\mathrm{N} 1, \mathrm{~N} 2, \mathrm{~N} 3, \mathrm{~N} 6$, and N8 subtypes (Webster and Govorkova, 2014). 
The H5 subtype includes the highly pathogenic (HPAIV) and the low pathogenic (LPAIV) avian influenza viruses, which belong to different genetic lineages [Influenza Research Database (IRD)]. The H5 HPAI viruses (arisen from A/goose/Guangdong/1/96(H5N1) - GsGDlineage) have been the focus of attention since 1997, when a respiratory infection caused by this virus subtype was diagnosed in humans for the first time. The highly pathogenic avian $\mathrm{H} 5 \mathrm{Nx}$ influenza viruses can be a severe danger for public health and the poultry industry. H5N8 HPAIV was first isolated from a domestic duck in China in 2010 and has continued to spread (Wu et al., 2014). Today, H5N6 has replaced $\mathrm{H} 5 \mathrm{~N} 1$ as the dominant HPAIV subtype in southern China, especially in ducks (Bi et al., 2016, Bulter et al., 2016; Lee et al., 2017; WHO, 2017). Two lineages of low pathogenic $\mathrm{H} 5$ influenza viruses have arisen from outside the GsGD lineage, Am-nonGsGD and EA-nonGsGD lineages. These lineages circulate among different species of wild ducks and other birds in American and Eurasian continents, respectively (IRD).

The surface glycoprotein, hemagglutinin (HA), is one of the main antigenic determinants, which induces antibody production in an infected organism. HA carries out two important functions in viral live cycle:(1) recognition and binding to cellular receptors and (2) mediation of the fusion of cellular and viral membranes to penetrate the host cell. The latter is dependent on the stability of a HA conformation at the $\mathrm{pH}$ of a given environment, termed "pH of fusion" (DuBois et al., 2011).

The HA of H5 influenza viruses isolated from humans, wild aquatic birds and chickens possess different properties (Matrosovich et al., 1999). However, immunization with a live waterfowl-origin avian influenza virus was able to effectively protect chickens against HPAIV (H5) infection (Crawford et al. 1998; Gambaryan et al., 2016). Therefore, increasing knowledge related to receptor-binding specificity, antigenic and immunogenic properties and variability of $\mathrm{H} 5$ viruses is essential for developing new antivirals and vaccines.

In our previous studies, we mapped the HA antigenic epitopes of an earlier mouse-adapted LPAI virus of American lineage (A/mallard/Pennsylvania/10218/84(H5N2) (Kaverin et al., 2002) and HPAI H5N1 human and avian viruses [(A/Vietnam/1203/04 and A/duck/Novosibirsk/56/05 (Kaverin et al., 2007; Rudneva et al., 2010)]. The present work aimed to map the HA epitopes of nonpathogenic A/duck/Moscow/4182/2010(H5N3) belonging to Eurasian nonGsGD lineage, thereby uncovering the HA antigenic epitopes for viruses of different phylogenetic lineages. For this purpose, we selected escape mutants using a set of monoclonal antibodies against HA and, for the first time, have sequenced the complete genome of escape mutants to estimate possible changes in viral proteins other than HA.

\section{Materials and Methods}

Viruses. Virus A/duck/Moscow/4182/2010(H5N3) (GenBank Acc. No. KF885672-KF885679) was kindly provided by A.S. Gambaryan (Chumakov Federal Scientific Center for Research and Development of Immune-and-Biological Products, Russian Academy of Sciences, Moscow, Russia). This virus was isolated from mallard feces collected on the shore of a pond in Moscow in October 2010. Virus variant A/duck/Moscow/4182-C/2010(H5N3) was obtained from A/duck/Moscow/4182/2010(H5N3) by means limiting dilution cloning in embryonated chicken eggs according to (Kaverin et al., 2002) and differed from the parent by two mutations, A188E and D264N, in HA. Viruses were propagated for $48 \mathrm{~h}$ in the allantoic cavities of 10-day-old embryonated chicken eggs at $37^{\circ} \mathrm{C}$ and stored $-80^{\circ} \mathrm{C}$ until use.

Monoclonal antibodies. A panel of virus-neutralizing MABs specific to the HA of $\mathrm{H} 5$ strains was used. MABs cp55, cp79, 176/26, 364/1 and 777/1 to A/chicken/Pennsylvania/8125/ 83(H5N2), and MABs VN04-10 and VN04-13 to the HA of A/Vietnam/1203/04(H5N1) influenza virus were generated in the Virology Department and the Department of Infectious Diseases, St Jude Children's Research Hospital (Memphis, USA). MAB 4F11 to A/duck/Novosibirsk/56/05(H5N1) was produced in N.F. Gamaleya National Research Center for Epidemiology and Microbiology D.I. Ivanovsky Instutute of Virology (Kushch et al., 2008).

Selection of escape mutants. Escape mutants have been obtained from A/duck/Moscow/4182-C/2010(H5N3) that was incubated with an excess of MAB for $1 \mathrm{~h}$ at room temperature followed by propagation in embryonated chicken eggs according to modification by Kaverin et al.(2002) of method by Webster and Laver (1980).

Nucleotide sequencing. During the process of selection and genetic engineering, HA gene structure was determined by Sanger sequencing as described earlier (Timofeeva et al., 2016). Finally, complete genome sequencing of the virus variant A/duck/Moscow/4182-C/2010(H5N3) and its escape mutants was performed by MiSeq Illumina platform as described (Voronina et al., 2018). Genomes were assembled using the CLC Genomics Workbench v. 9.5.2 and 12.0. Genome sequences were deposited in GenBank with Acc. Nos. MF992939-MF993018 and MK357950-MK358013 (Table 1).

Hemagglutination and hemagglutination inhibition tests (HI) were performed using $0.75 \%$ chicken erythrocytes by a standard method (Palmer et al., 1975).

Assessment of HA thermostability. Viruses present in clarified allantoic fluid were diluted to $128 \mathrm{HA}$ units and incubated in phosphate-buffered saline (PBS) for $40 \mathrm{~min}$ at temperatures ranging from $45^{\circ} \mathrm{C}$ to $70^{\circ} \mathrm{C}$ in a Mastercycler nexus gradient thermostat (Eppendorf, Germany). After incubation, viruses were titrated by HA assay with $0.75 \%$ erythrocytes. The temperature, which resulted in a decrease of $6 \log _{2}$ units in the HA 


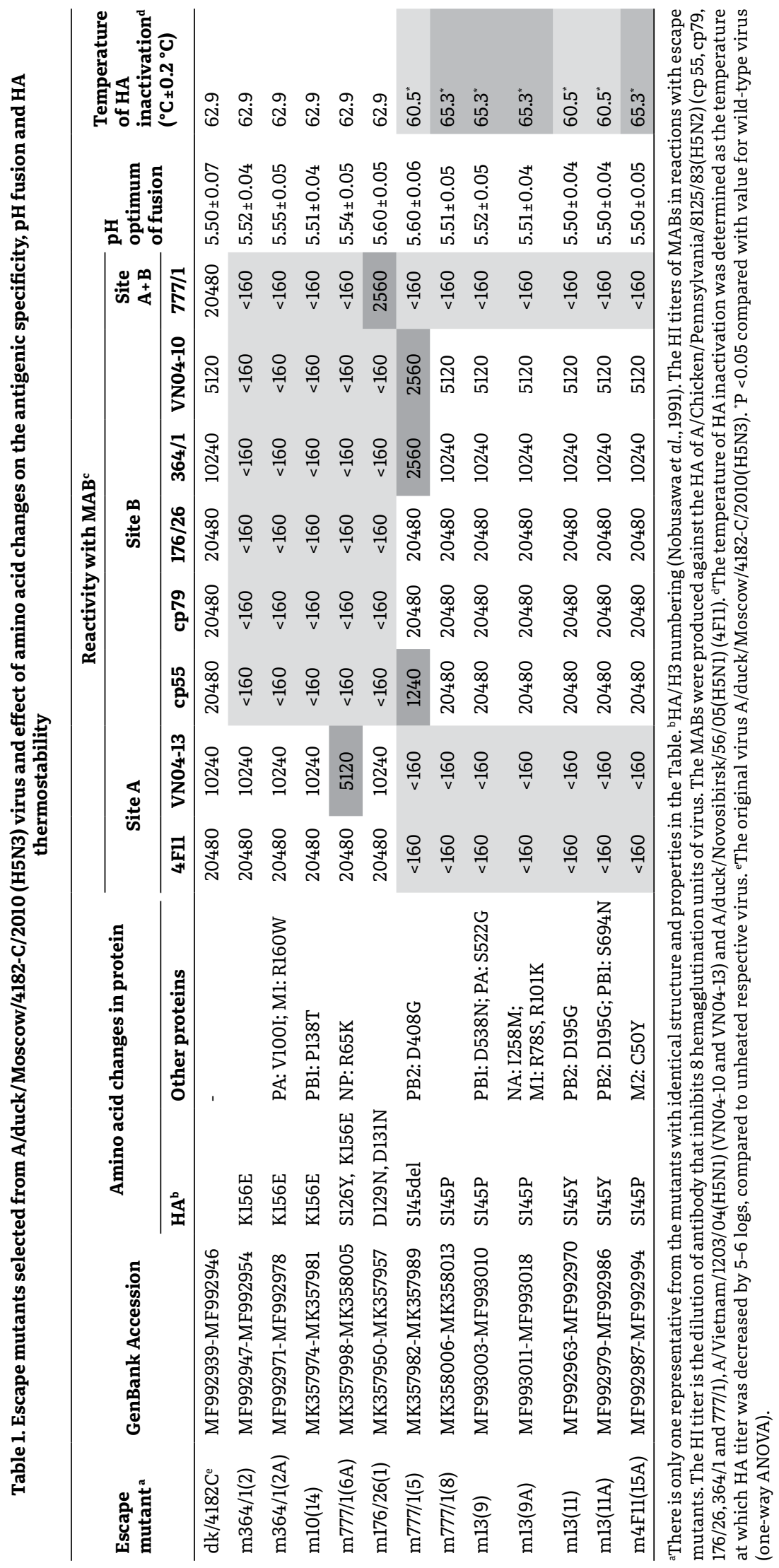


titer, was taken as the temperature of HA heat inactivation (Rudneva et al., 2013).

Assessment of pH optimum of fusion. Viruses present in the clarified allantoic fluid $(250 \mu \mathrm{l})$ were diluted to $128 \mathrm{HA}$ units in PBS, were mixed with $50 \mu$ l of $2.5 \%$ suspension of chicken erythrocytes and incubated on ice for $1 \mathrm{~h}$ to allow virus binding (Krenn et al., 2011). After centrifugation at $72 \mathrm{~g}$, pellets were resuspended in $250 \mu \mathrm{l}$ of 2 -(N-morpholino)ethanesulfonic acid (MES) buffer at various $\mathrm{pH}$ values (from 5.0 to 6.5 ) and incubated at $37^{\circ} \mathrm{C}$ for $1 \mathrm{~h}$. Then, samples were precipitated $(72 \mathrm{~g})$ and supernatants $(170 \mu \mathrm{l})$ were transferred to 96 -well plates to determine the optical density (at $450 \mathrm{~nm}$ ) which correlates with the erythrocyte hemolysis induced by virus and cell membrane fusion. The lowest $\mathrm{pH}$ value that resulted in the highest optical density was taken as the optimal $\mathrm{pH}$. Reported results are the means of $\mathrm{pH}$ of fusion $\pm \mathrm{SD}$ measured in 3 replicative experiments (Rudneva et al., 2013).

Virus growth kinetics in embryonated chicken eggs. 10-dayold embryonated chicken eggs were infected with 1000 50\% egg infectious dose $\left(\operatorname{EID}_{50}\right)$ of tested viruses and incubated for $12,24,36$ and $48 \mathrm{~h}$ at $37^{\circ} \mathrm{C}$. After each time interval, a group of 5 eggs were inoculated with the same virus, then transferred to $4^{\circ} \mathrm{C}$ to be chilled overnight. Allantois fluids were titrated by hemagglutination assay and the data are expressed as mean $\log _{2}$ reciprocal HA titer \pm SD.

Mutant virus recovery. Viruses with definite mutations in HA were created on the base of VN-H5N1-PR8/CDC-RG virus by reverse genetics, as described earlier (Hoffmann et al., 2000; Timofeeva et al., 2016). Briefly, nucleotide substitutions were introduced into plasmids carrying the HA gene using the QuikChange XL Site-Directed Mutagenesis Kit (Stratagene, United States). Plasmid transfection was performed in a mixed culture of HEK-293T-MDCK for virus recovery. Virus was subsequently propagated in 10-day-old chicken embryos for $48 \mathrm{~h}$ at $37^{\circ} \mathrm{C}$.

Statistical analysis. Analysis of variance (ANOVA) was used for comparing the values of $\mathrm{pH}$ of fusion, temperatures of $\mathrm{HA}$ inactivation, and HI assay. A probability value equal to 0.05 was used to indicate that the received data were not a result of chance.

\section{Results}

Selection and antigenic characterization of H5N3 escape mutants

Virus A/duck/Moscow/4182-C/2010(H5N3) was used as the wild-type virus for the selection of escape mutants. Primarily, antigenic properties were tested in cross-reaction with MAB panels against HA of strains A/chicken/ Pennsylvania/8125/83(H5N2), A/Vietnam/1203/04(H5N1) and A/duck/Novosibirsk/56/05(H5N1) (Kaverin et al.,
2002, 2007; Kushch et al., 2008). Five positive reactions were observed from 7 MABs against A/chicken/ Pennsylvania/8125/83(H5N2), 2 from 7 MABs against A/ Vietnam/1203/04(H5N1) and only 1 from 6 MABs against A/duck/Novosibirsk/56/05(H5N1) (data not shown). The MABs, which positively reacted with A/duck/ Moscow/4182-C/2010(H5N3), were used to obtain escape mutants (Table 1, Fig. 1). These previously characterized MABs could be divided based on their specificity for HA antigenic sites: MABs recognizing site A (4F11, VN04-13), MABs specific for site B (cp55, cp79, 176/26, 364/1, and VN04-10) and MAB 777/1 that recognizes overlapping A and $B$ sites.

17 escape mutants were selected due to the neutralizing ability of MABs and their antigenic properties were tested by HI assay (Table 1). Results suggest that no escape mutants cross-reacted with MAB 777/1, except mutant m176/26(1). Three escape mutants (m364/1(2), m364/1(2A) and $\mathrm{m} 10(14))$ retained reactivity with site $A$-specific MABs and 7 others (m777/1(5), m777/1(8), m13(9), m13(9A), m13(11), $\mathrm{m} 13(11 \mathrm{~A})$ and $\mathrm{m} 4 \mathrm{~F} 11(15 \mathrm{~A}))$ with site B-specific MABs.

\section{Sequence analysis of H5N3 escape mutants}

Complete genomes of 17 escape mutants were sequenced. All mutants contained 1 or 2 amino acid changes in HA. Nine escape mutants carried additional mutations in viral proteins other than HA (NA, PA, PB1, PB2, M1, M2 and NP, Table 1).

Disruption of immune reactivity with site A-specific MABs correlated with amino acid changes in position 145 of HA by polymorphisms (S, P, Y, del). Mutation K156E was observed in escape mutants, which displayed altered reactivity with site B-specific MABs. One of those mutants - m777/1(6A) - contained an additional mutation in HA (K156E+S126Y).

Mutant m176/26(1) acquired two mutations, HA: $\mathrm{D} 129 \mathrm{~N}+\mathrm{D} 131 \mathrm{~N}$, and retained reactivity with site A-specific MABs (4F11 and VN04-13) and 777/1 recognizing both antigenic sites $\mathrm{A}$ and $\mathrm{B}$.

Since antigenically important mutations might possess pleiotropic effects that would give mutants the advantage for predominant spread (Rudneva et al., 2013), we tested some phenotypic features of escape mutants.

Estimation of $\mathrm{pH}$ of fusion and HA thermostability of H5N3 escape mutants

The erythrocyte hemolysis test showed that the optimal $\mathrm{pH}$ of fusion for escape mutants was not different than that of wild-type virus (Table 1 ).

Differences in HA thermostability were observed for mutants with varying amino acids at position 145 . Viruses 
(a)

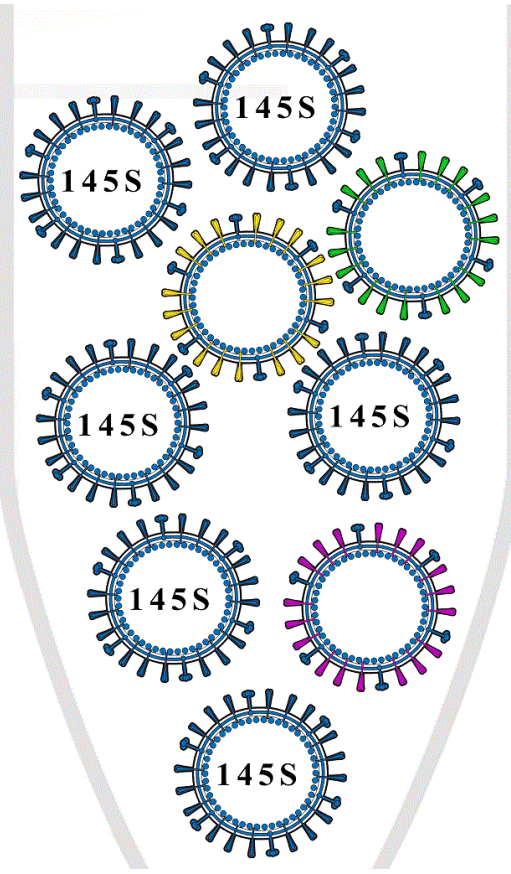

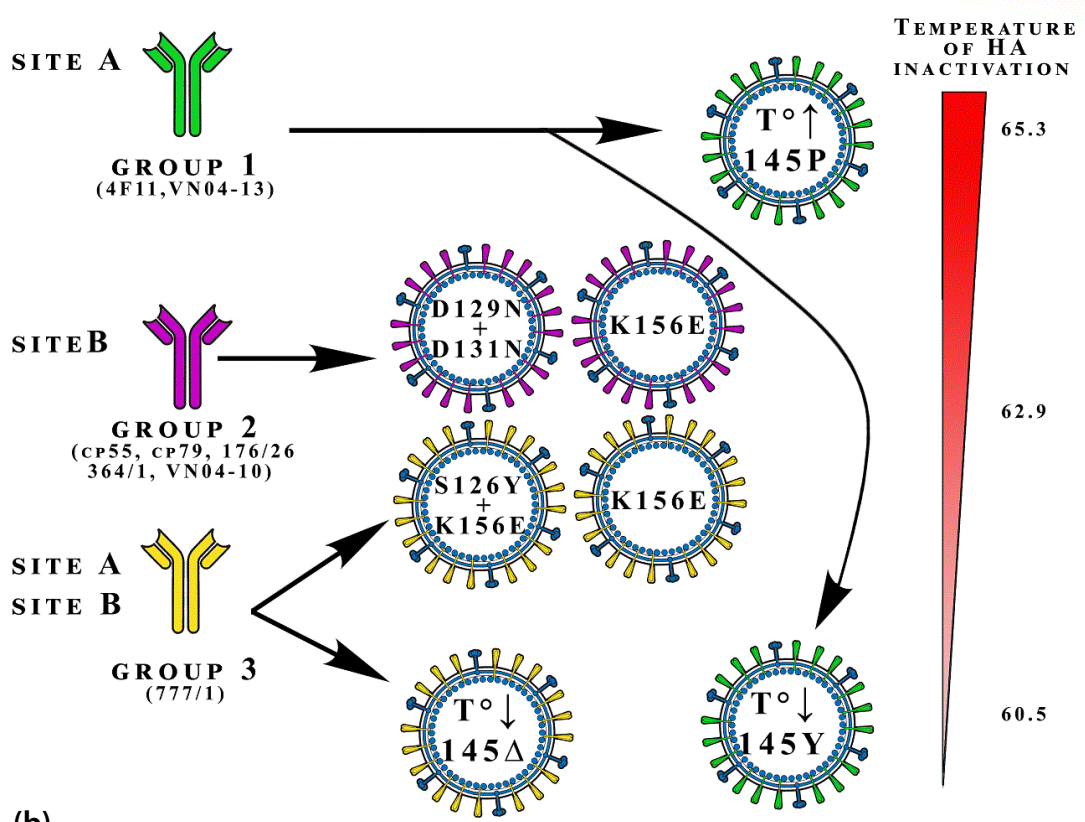

Fig. 1

Selection of LPAIV A/duck/Moscow/4182-C/2010 (H5N3) escape mutants by specific MABs

(a) Due to spontaneous mutations during replication, existing heterogenic population of $\mathrm{H} 5$ influenza virus contains predominant $145 \mathrm{~S}$ in HA and minor polymorphic mutations (not marked). HA specific MABs neutralize the virions with appropriate epitope except for virions with minor mutations that changed epitope and allowed virus to survive. Mutations are designated inside virions. The MABs and complementary HA antigenic sites on the viral surface are colored - site A (green), site B (purple), and site A+B (yellow). (b) Point mutation at position 145 in HA promotes an escape from MABs action and changes the virus thermostability.

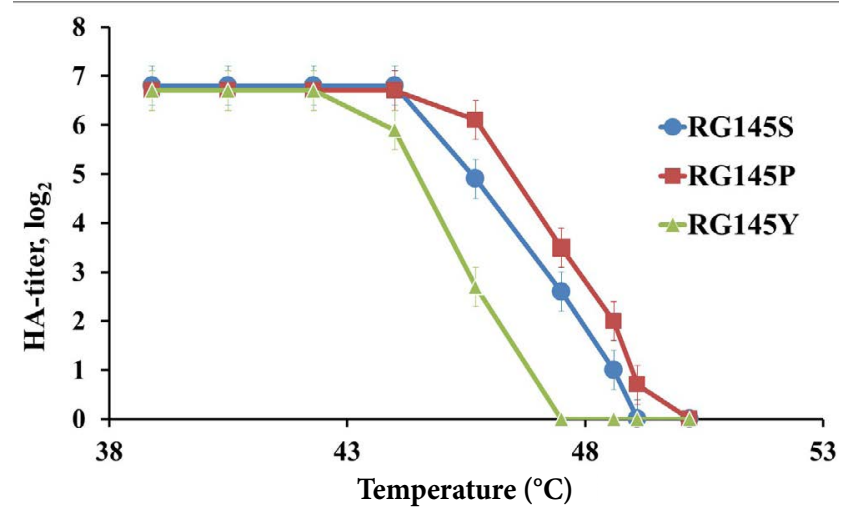

Fig. 2

Thermostability of recombinant VN-H5N1-PR8/CDC-RG viruses with mutation in position 145 of $\mathrm{HA}$ after incubation at the indicated temperatures for $\mathbf{4 0} \mathrm{min}$

Hemagglutination titers are shown on the $Y$ axis.

carrying mutation $145 \mathrm{del}(\mathrm{m} 777 / 1(5))$ or $145 \mathrm{Y}$ (m13(11) and $\mathrm{m} 13(11 \mathrm{~A}))$ demonstrated statistically significant decreases in HA heat inactivation $\left(60.5 \pm 0.2^{\circ} \mathrm{C}, \mathrm{P}<0.05\right)$ compared to wild-type. In contrast, escape mutants containing $145 \mathrm{P}$ (m777/8, m13(9), m13(9A), and m4F11(15A)) exhibited a significantly elevated temperature $\left(65.3 \pm 0.2^{\circ} \mathrm{C}, \mathrm{P}<0.05\right)$ of HA heat inactivation compared to the wild-type $\left(62.9 \pm 0.2^{\circ} \mathrm{C}, \mathrm{P}<0.05\right)$. The other escape mutants did not have remarkable changes relative to the initial virus A/duck/Moscow/4182-C/2010(H5N3).

Generation of viruses by reverse genetics and analysis of their thermostability

To uncover the role of amino acid 145 in maintenance of HA thermostability, 3 virus variants were created by reverse genetics of $\mathrm{VN}-\mathrm{H} 5 \mathrm{~N} 1-\mathrm{PR} 8 / \mathrm{CDC}-\mathrm{RG}$ vaccine strain. They differed only by the single mutation in HA: RG145S, RG145P and RG145Y. As shown in Fig. 2, mutant RG145P possessed the highest thermostability of the mutants, while the other 2 mutants remained comparable to escape mutants.

Surprisingly, we observed remarkable differences (about $12^{\circ} \mathrm{C}$ ) in average temperature of HA thermostability between initial avian virus A/duck/Moscow/4182C/2010(H5N3) and human VN-H5N1-PR8/CDC-RG. This result can be explained by host-dependent origin and the specific properties of these viruses, which are caused by viral structure. 
(a)

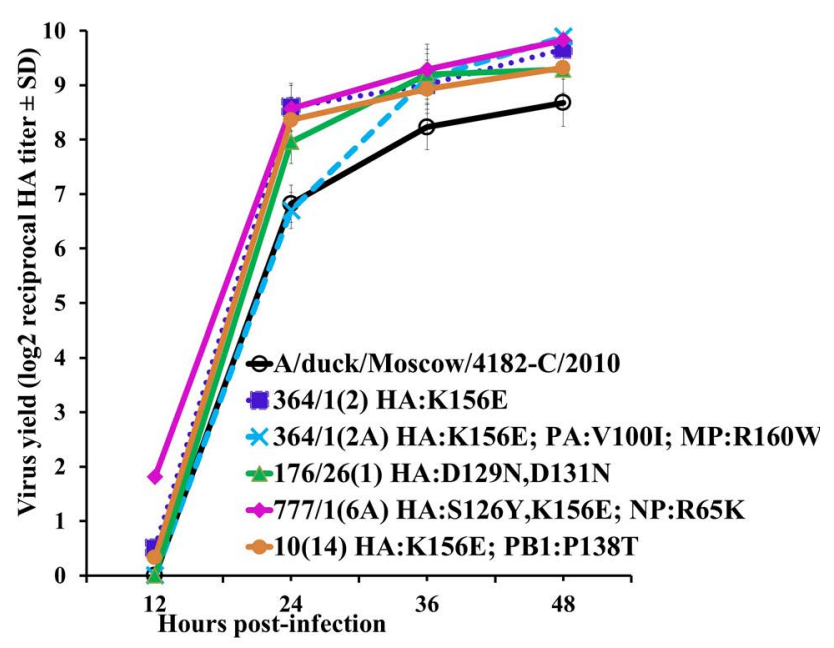

(b)

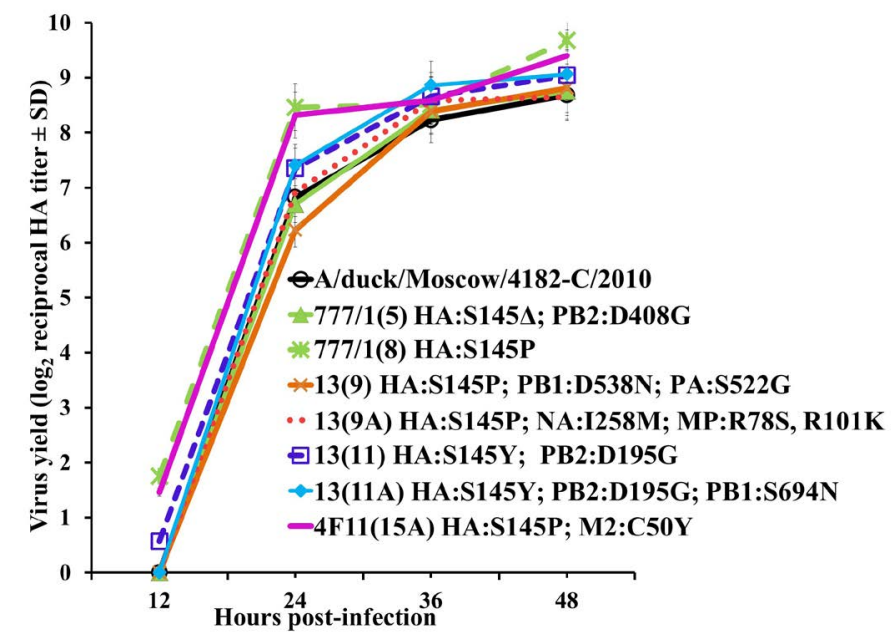

Fig. 3

Replication kinetics of H5N3 escape mutants in comparison with wild-type virus A/duck/Moscow/4182-C/2010 in embryonated chicken eggs

Eggs were infected with viruses at $1000 \mathrm{EID}_{50}$.Virus yield ( $\log _{2}$ reciprocal HA titer) was titrated by HA assay $12,24,36$ and $48 \mathrm{~h}$ post-infection. Each data point represents the mean value from 3 independent experiments.

\section{Virus replication in embryonated chicken eggs}

All escape mutants carrying HA: K156E substitution had a similar replication pattern and gave $2-4$ times greater virus yield after 36 and $48 \mathrm{~h}$ post-infection (h.p.i.) than the parent strain. The exception was mutant m777/1(6A) (HA: K156E+S126Y, NP: R65K). This mutant possessed additional mutations in HA and NP and demonstrated increased virus replication in the first 12 h.p.i (Fig. 3a).

Similar increased replication, in the first 12 h.p.i., was observed for mutants with a single substitution, HA: S145P (m777/1(8)) and those with an additional mutation in the M2 protein (m4F11(15A); HA: S145P+M2: C50Y). Other mutants carrying HA: 145P and additional mutations in internal proteins did not have this property but had growth curves similar to that of the parent virus, A/duck/Moscow/4182-C/2010(H5N3) (Fig. 3b).

\section{Comprehensive analysis of mutations}

Comprehensive analysis was performed for each position, in which mutations had occurred. The technical tools from the Influenza Research Database website (https:// www.fludb.org) were applied. We explored the occurrence frequency of substituted amino acids in natural isolates, their polymorphisms and locations relative to functional sites and epitopes that were predicted or experimentally determined (Supplementary information (SI) S1-S6).
Antigenically significant mutations at 126, 129, 131, 145 and 156 positions of HA coincided with short linear epitopes (SI S1-S3) and were mapped to the 3-dimensional structure of HA/H5(Fig.4) in our previous studies (Kaverin et al., 2002, 2007, Rudneva et al., 2010). There was some amino acid variety in the positions mentioned above where, among natural $\mathrm{H} 5$ isolates, $126 \mathrm{~S}$ and $156 \mathrm{~K}$ were the prevailing mutations observed. Two amino acids, Serine and Proline, dominated in position 145. Predominance of amino acids depends on viral host species specificity or the influence of immune pressure. Thus, differences in prevailing amino acids at positions 129 and 131 were observed between H5N1 viruses of the GsGD lineage (129D and 131E) and non-pathogenic wild duck viruses of other lineages, Am-nonGsGD and EA-nonGsGD (129N and 131D).

Among the original viruses (A/mallard/Pennsylvania/10218/84, A/Vietnam/1203/04, A/duck/Novosibirsk/56/05 and A/duck/Moscow/4182-C/2010) that were used to obtain escape mutants (Kaverin et al., 2002, 2007; Rudneva et al.,2010), the common identical immunodominant amino acids in HA were 126S, 145S, and 156K (SI S3). Moreover, those viruses possessed identical short linear epitopes (145-SSFFRNVVWLIKK-157 (H3 numbering); corresponds to 157-169 HA/H5 precursor numbering).

Escape mutants that survived the addition of HAspecific MABs possessed substitutions with an alternative amino acid in the corresponding positions (SI S1, S2), except the m777/1(5), which contained a 145del (HA: S145del, PB2: D408G). No viruses with this deletion have 


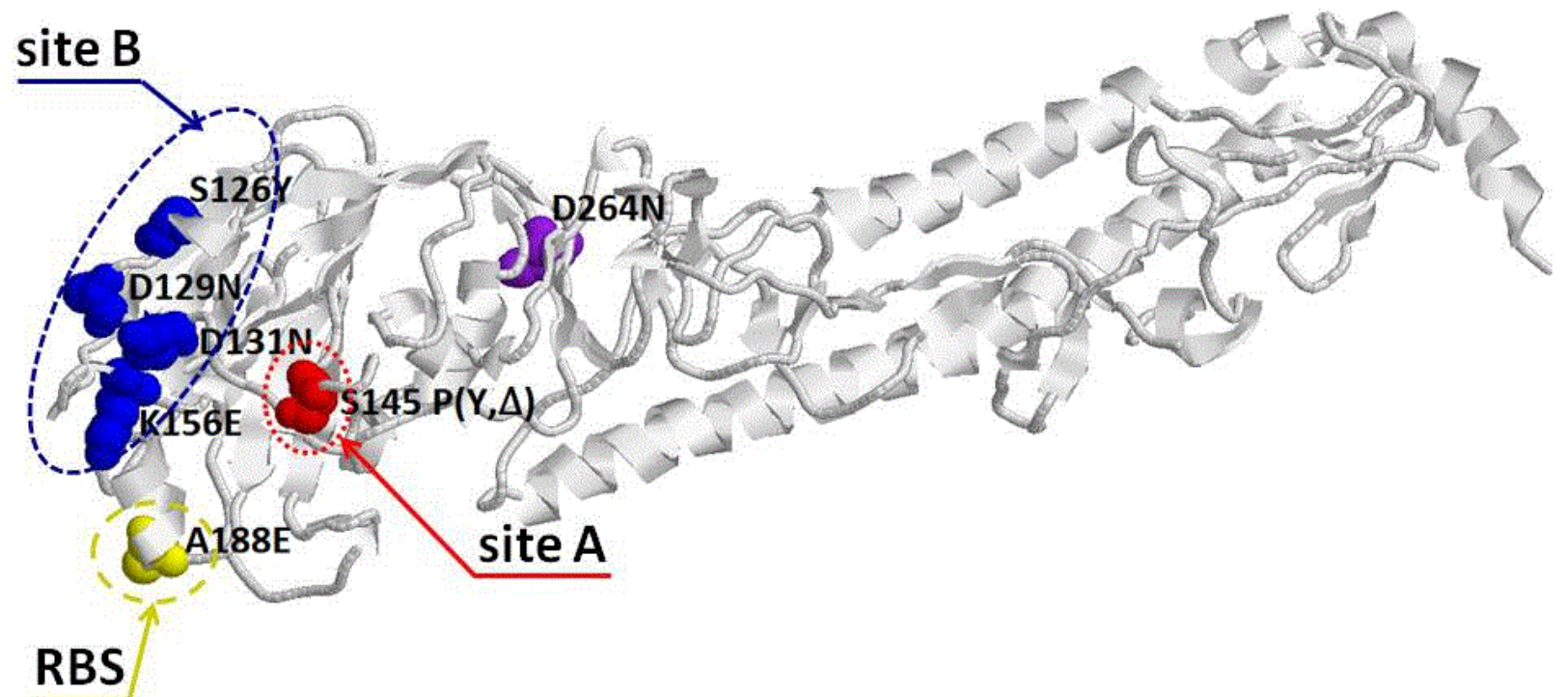

Location of amino acid changes in the HA of $\mathrm{H} 5$ escape mutants in the 3-dimensional structure of A/Vietnam/1203/2004 (Stevens et al., 2006)

Antigenic site A (in $\mathrm{HA} / \mathrm{H} 3$ ) is colored in red; antigenic site B (in HA/H3) is colored in blue. Mutations differing between cloned variant A/duck/Moscow/4182-C/10 and the original strain A/duck/Moscow/4182/2010(H5N3 are colored in yellow and purple. RBS - receptor binding site. Image was created with RasMol (www.rasmol.org) and the HAl structure was obtained from the RCSB Protein Data Bank (PDB ID 2FKO) using pdb-tools software (https://doi.org/https:doi.org/10.2210/pdb2FKO/pdb). Amino acid positions are designated in mature H3 numbering.

been found in nature and reverse genetics failed to produce a viable 145 del virus.

Proline, at position 145 , has a conformationally inflexible structure, which may increase the HA protein conformational stability at elevated temperatures (PeralvarezMartin et al., 2008), as was shown by reverse genetics in this work.

The location of residue 145 coincides with antigenic site $A$ in the lateral loop near the receptor binding site (Fig. 4). A mutation in this position may lead to conformational changes and may affect receptor specificity of those mutated influenza viruses. Indeed, the mutation S145P resulted in increased affinity towards sialyl receptors, as was shown earlier (Ilyushina et al., 2004; Santos et al., 2019). Resistance to high temperatures and increased affinity for cell receptors can give viruses an advantage of more successful environmental spread. In fact, it should be noted that the occurrence frequency of the S145P substitution among $\mathrm{H} 5$ viruses increased from $8.9 \%$ to $87.8 \%$ between 2010-2019 (SI S4). However, this effect may be due to vaccination against HPAIV H5N1.

Residue 156 lies at the tip of the globular head of the HA molecule (Fig. 4). Mutation K156E did not affect HA thermostability but increased virus replicative capacity in comparison with wild-type (Fig.3b). The involvement of the polymorphism at residue 156 in altering host-depend- ent antigenic properties and reproductive capacities was described earlier (Katz et al., 1987; Kilbourne et al., 1988; Gambaryan et al.,1998). The charge of amino acids in this position influence electrostatic interactions between the globular part of viral HA and the negatively charged cell surface. Replacement K156E resulted in the wild-type amino acid charge to flip from positive to negative, thereby reducing the binding affinity between the virus and the cell membrane. Thus, newly generated viral particles could be released more easily from the cell surface and spread with fluid.

In addition to mutations in HA, escape mutants have been found to have concomitant mutations in internal proteins. All mutations are located in experimentally determined epitopes in positions that have also been characterized by polymorphisms. Most of these epitopes are highly conservative. We studied 12 short linear epitopes (8-15 a.a. in length) of PB2, PB1, PA, NP, M1, M2, and NA proteins for A/duck/Moscow/4182-C/2010, A/Vietnam/1203/04 and A/duck/Novosibirsk/56/05. Among them, 8 epitopes of these viruses have the same structure (PB2: 186-200, 406-411; PB1: 135-143, 538-546; PA: 515-523; NP: 61-68; M1: 76-84; M2: 45-53) (SI S5, S6).

Here, escape mutants acquired point mutations, in which amino acids were substituted by predominant (PB1: S694N, M1: R101K) or alternative ones (PB2: D195G, 
M2: C50Y), which have been seen previously in natural isolates, apart from M1: R160W, which was not found in nature. Some mutations are located on a linear sequence within functional domains that provide protein-protein or RNA-protein interaction. Judging by the lack of significant changes in the reproduction of escape mutants relative to the wild-type virus (Fig. 3a,b), it is unlikely that these substitutions have a functional significance. Additionally, as is known, substitutions found in internal proteins of escape mutants are less frequent.

\section{Discussion}

Viruses circulating in natural habitats undergo immune pressure from host organisms that have had previous contact with those influenza viruses. However, some viral particles can escape this immune pressure and continue their existence and spread due to advantageous mutations. We have simulated this immune selection process in the laboratory by using monoclonal antibodies against HA to produce escape mutants. Additionally, a similar approach allowed us to map the fine antigenic structure of H5N1 HPAIV (Kaverin et al., 2007; Rudneva et al., 2010). In this study, we investigated nonpathogenic virus A/duck/ Moscow/4182-C/2010 to determine the common epitopes between LPAI and H5N1 HPAI viruses.

Influenza viruses can spontaneously mutate during replication and produce heterogenic populations with mutations in different genome segments and viral proteins. These events can explain polymorphisms existing at some positions of viral proteins. However, only a restricted number of mutants can predominantly spread in particular conditions. In our case, all escape mutants with HA substitutions arose from immune pressure mediated by the action of monoclonal antibodies, which were specific against HA (Fig. 1). Therefore, depending on existing heterogenic population and MAB specificity, varying amino acids at immunodominant positions of escape mutants may occur.

For the first time, the complete genomes of escape mutants were sequenced in this work. The character of mutations that were found in proteins other than HA suggests their sporadic occurrence. Mainly, the mutations that occurred were represented by substitutions with predominant or alternative amino acids existing in the nature.

However, we hypothesized that some concomitant mutations in internal proteins were induced by defective changes in HA, such as deletion $145 \mathrm{del}$ or the rare mutation $145 \mathrm{Y}$, in order to compensate this defect. For example, mutants m777/1(5) (HA: S145del; PB2: D408G), m13(11) (HA: S145Y; PB2: D195G) and m13(11A) (HA: S145Y;
PB2: D195G; PB1: S694N) had a level of virus accumulation that did not differ from wild-type virus even with defective mutations in HA.

Therefore, we determined that the general immunodominant positions 126, 145 and 156 of HA in H5 viruses belong to the 3 different phylogenetic lineages GsGD, Am-nonGsGD, and EA-nonGsGD. These are located in the antigenic sites A (145) and B (126 and 156) on the 3-dimensional structure of HA.

Moreover, representatives of the highly pathogenic gs-GD- and low pathogenic EA-nonGD- lineages have the same structure of short linear epitopes in HA (145-157) and internal proteins (PB2:186-200,406-411; PB1:135-143, 538-546; PA:515-523; NP:61-68; M1:76-84; M2: 45-53). This information contributes an explanation of the protective effect of the mallard's non-pathogenic virus A/duck/ Moscow/4182/2010(H5N3) (EA-nonGD) against challenge with the highly pathogenic H5N1 virus gs-GD, as was shown in chickens [Gambaryan et al, 2016].

Acknowledgments. We are especially grateful to Dr. R. Webster (St. Jude Children's Research Hospital,Memphis, United States) for kindly provided system of eight plasmids for recovery of VNH5N1-PR8/CDC-RG virus; to Dr. A.S. Gambaryan for the gift of A/duck/Moscow/4182/2010(H5N3) virus; to Dr. R. Webster and Dr. E. A. Govorkova for providing the panel of MAbs to the HA of $\mathrm{H} 5$ strains. We are especially grateful to Dr. N.A. Ilyushina for helpful discussions.

Supplementary information is available in the online version of the paper.

\section{References}

Bi Y, Chen Q, Wang Q, Chen J, Jin T, Wong G, Quan C, Liu J, Wu J, Yin R, Zhao L, Li M, Ding Z, Zou R, Xu W, Li H, Wang H, Tian K, Fu G, Huang Y, Shestopalov A, Li S, Xu B, Yu H, Luo T, Lu L, Xu X, Luo Y, Liu Y, Shi W, Liu D, Fu G (2016): Genesis, evolution and prevalence of H5N6 avian influenza in China. Cell Host \& Microbe 20(6), 810-821. https://doi.org/10.1016/j.chom.2016.10.022

Bulter J, Stewart CR, Layton DS, Phommachanh P, Harper J, Payne J, Evans RM, Valdeter S, Walker S, Harvey G, Shan S, Bruce MP, Rootes CL, Gough TJ, Rohringer A, Peck GR, Fardy SJ, Karpala AJ, Johnson D, Wang J, Douangngeun B, Morrissy G, Wong FYK, Bean AGD, Bingham J, Williams DT (2016): A novel reassortant H5N6 influenza A virus from Lao People's Democratic Republic is highly pathogenic in chickens. Plos One 11(9), e0162375. https://doi.org/10.1371/iournal.pone.0162375

Crawford JM, Garcia M, Stone H, Swayne D, Slemons R, Perdue ML (1998): Molecular characterization of the hemagglutinin gene and oral immunization with a waterfowl-origin avian influenza virus. Avian Dis. 42(3), 486-496. https://doi.org/10.2307/1592675 
DuBois RM, Zaraket H, Reddivari M, Heath RJ, White SW, Russell CJ (2011): Acid stability of the hemagglutinin protein regulates H5N1 influenza virus pathogenicity. PLoS Pathogens 7(12), e1002398. https://doi.org/10.1371/ journal.ppat.1002398

Gambaryan AS, Boravleva EY, Lomakina NF, Kropotkina EA, Gordeychuk IV, Chvala IA, Drygin VV, Klenk H-D, Matrosovich MN (2016): Immunization with live nonpathogenic H5N3 duck influenza virus protects chickens against highly pathogenic $\mathrm{H} 5 \mathrm{~N} 1$ virus. Acta Virol. 60, 316-327. https://doi.org/10.4149/av_2016_03_316

Gambaryan AS, Matrosovich MN, Bender CA, Kilbourne ED (1998): Differences in the biological phenotype of low-yielding $(\mathrm{L})$ and high-yielding $(\mathrm{H})$ variants of swine influenza virus $\mathrm{A} / \mathrm{NJ} / 11 / 76$ are associated with their different receptor-binding activity. Virology 247, 223-231. https://doi.org/10.1006/viro.1998.9274

Hoffmann E, Neumann G, Kawaoka Y, Hobom G, Webster RG (2000): A DNA transfection system for generation of influenza A virus from eight plasmids. Proc. Natl. Acad. Sci. USA 97, 6108-6113. https://doi.org/10.1073/ pnas.100133697

Ilyushina NA, Rudneva IA, Gambaryan AS, Tuzikov AB, Bovin NV (2004): Receptor specificity of H5 influenza virus escape mutants. Virus Res.100(2),237-241. https://doi. org/10.1016/j.virusres.2003.12.032

IRD Highly Pathogenic H5 Clade Classification Tool January 14,2015. https://www.fludb.org/brcDocs/documents/ IRD_H5_CLADE_SOP.pdf

Katz JM, Naeve CW, Webster RG (1987): Host cell-mediated variation in H3N2 influenza viruses. Virology 156(2), 386-395.https://doi.org/10.1016/0042-6822(87)90418-1

Kaverin NV, Rudneva IA, Govorkova EA, Timofeeva TA, Shilov AA, Kochergin-Nikitsky KS, Krylov PS, Webster RG (2007): Epitope mapping of the hemagglutinin molecule of a highly pathogenic H5N1 influenza virus by using monoclonal antibodies. J. Virol. 81, 12911/12917. https://doi.org/10.1128/JVI.01522-07

Kaverin NV, Rudneva IA, Ilyushina NA, Varich NL, Lipatov AS, Smirnov YA, Govorkova EA, Gitelman AS, Lvov DK, Webster RG (2002): Structure of antigenic sites on the haemagglutinin molecule of $\mathrm{H} 5$ influenza virus and phenotypic variation of escape mutants. J. Gen. Virol. 83,2497-2505.https://doi.org/10.1099/0022-131783-10-2497

Kilbourne ED, Taylor AH, Whitaker CW, Sahai R, Caton A (1988): Hemagglutinin polymorphism as the basis for lowand high-yield phenotypes of swine influenza virus. Proc. Natl. Acad. Sci. USA 85, 7782-7785. https://doi. org/10.1073/pnas.85.20.7782

Krenn BM, Egorov A, Romanovskaya-Romanko E, Wolschek M, Nakowitsch S, Ruthsatz T, Kiefmann B, Morokutti A, Humer J, Geiler J, Cinatl J, Michaelis M, Wressnigg N, Sturlan S, Ferko B, Batishchev OV, Indebom AV, Zhu R, Kastner M, Hinterdorfer P, Muster T, Romanova J (2011): Single HA2 mutation increases the infectivity and immunogenicity of a live attenuated H5N1 intranasal influenza vaccine candidate lacking NS1.
PloS One 6, e18577. https://doi.org/10.1371/journal. pone.0018577

Kushch AA, Klimova RR, Masalova OV, Fedorova NE, Botikov AG, Fedyakina IT, Burtseva EI, Isaeva EI, Deryabin PG, Lvov DK (2008): Monoclonal antibodies to highly pathogenic avian influenza $\mathrm{A}(\mathrm{H} 5 \mathrm{~N} 1)$ strain isolated in the Russian Federation: Development and properties. Vopr. Virusol. 53(5), 9-14 (in Russian).

Lee D-H, Bertran K, Kwon J-H, Swayne DE (2017): Evolution, global spread, and pathogenicity of highly pathogenic avian influenza H5Nx clade 2.3.4.4. J. Vet. Sci. 18(51), 269-280. https://doi.org/10.4142/jvs.2017.18.S1.269

Matrosovich MN, Zhan N, Kawaoka Y, Webster R (1999): The surface glycoproteins of $\mathrm{H} 5$ influenza viruses isolated from humans, chickens, and wild aquatic birds have distinguishable properties. J. Virol. 73, 1146-1155. https://doi.org/10.1128/JVI.73.2.1146-1155.1999

Nobusawa E, Aoyama T, Kato H, Suzuki Y, Tateno Y, Nakajima K (1991): Comparison of complete amino acid sequences and receptor-binding properties among 13 serotypes of hemagglutinin of influenza A viruses. Virology 182(2), 475-485. https://doi.org/10.1016/0042-6822(91)90588-3

Palmer DF, Dowdle WR, Coleman MT and Schild GC (1975): Advanced laboratory techniques for influenza diagnosis. U.S. Department of Health, Education, and Welfare, immunology series no.6. Centers for Disease Control, Atlanta, GA.

Peralvarez-Martin A, Lorenz-Fonfria VA, Simon-Vazquez R, Gomariz M, Mesequer I, Querol E, Padros E (2008): Influence of proline on the thermostability of the active site and membrane arrangement of transmembrane protein. Biophys. J. 95(9), 4384-4395. https://doi. org/10.1529/biophysj.108.136747

Rudneva IA, Kushch AA, Masalova OV, Timofeeva TA, Klimova RR, Shilov AA, Ignatieva AV, Krylov PS and Kaverin NV (2010): Antigenic Epitopes in the Hemagglutinin of Qinghai-Type Influenza H5N1 Virus. Viral Immunol. 23(2), 181-187. https://doi.org/10.1089/vim.2009.0086

Rudneva IA, Timofeeva TA, Ignatieva AV, Shilov AA, Krylov PS, Ilyushina NA, Kaverin NV (2013): Pleiotropic effects of hemagglutinin amino acid substitutions of $\mathrm{H} 5$ influenza escape mutants. Virology 447, 233-239. https:// doi.org/10.1016/j.virol.2013.09.013

Santos JJS, Abente EJ, Obadan AO, Thompson AJ, Ferreri L, Geiger G, Gonzalez-Reiche AS, Lewis NS, Burke DF, Rajão DS, Paulson JS, Vincent AL, Perez DP (2019): Plasticity of amino acid residue 145 near the receptor binding site of $\mathrm{H} 3$ swine influenza A viruses and its impact on receptor binding and antibody recognition. J. Virol. 93(2), e01413-18. https://doi.org/10.1128/JVI.01413-18

Stevens J, Blixt O, Tumpey TM, Taubenberger JK, Paulson JC, Wilson IA (2006): Structure and receptor 50 the hemagglutinin from an H5N1 influenza virus. Science 312, 404-410. https://doi.org/10.1126/science.1124513

Timofeeva TA, Sadykova GK, Rudneva IA, Boravleva EY, Gambaryan AS, Lomakina NF, Mochalova LV, Bovin NV, Usachev EV, Prilipov AG (2016): Changes in the phenotypic properties of highly pathogenic influenza A 
virus of H5N1 subtype induced by N186I and N186T point mutations in hemagglutinin. Mol. Biol. 50(5), 755-761. https://doi.org/10.1134/S0026893316050174 Voronina OL, Ryzhova NN, Aksenova EI, Kunda MS, Sharapova NE, Fedyakina IT, Chvala IA, Borisevich SV, Logunov DY, Gintsburg AL (2018): Genetic features of highly pathogenic avian influenza viruses A(H5N8), isolated from the European part of the Russian Federation. Infect. Genet. Evol. 63, 144-150. https://doi.org/10.1016/i. meegid.2018.05.022

Webster RG, Govorkova EA (2014): Continuing challenges in influenza. Ann. N.Y. Acad. Sci. 1323, 115-139. https:// doi.org/10.1111/nyas.12462
Webster RG, Laver WG (1980): Determination of the number of nonoverlapping antigenic areas on Hong Kong (H3N2) influenza virus hemagglutinin with monoclonal antibodies and the selection of variants potential epidemiological significance. Virology 104, 139-148. https://doi.org/10.1016/0042-6822(80)90372-4

World Health Organization. Situation updates - Avian influenza (2017): https://www.who.int/influenza/human_animal_interface/avian_influenza/archive/ru/

Wu H, Peng X, Xu L, Jin C, Cheng L, Lu X, Xie T, Yao H, Wu N (2014): Novel reassortant influenza A (H5N8) viruses in domestic ducks, eastern China. Emerg. Infect. Dis. 20(8),1315-1318. https://doi.org/10.3201/eid2008.140339 